\title{
Editorial Overview: \\ Positive Affect: Taxonomies, Mechanisms and Applications
}

\author{
Henk van Steenbergen ${ }^{1,2}$, Disa Sauter ${ }^{3}$, Blair Saunders ${ }^{4} \&$ Gilles Pourtois ${ }^{5}$ \\ ${ }^{1}$ Leiden Institute for Brain and Cognition, the Netherlands \\ ${ }^{2}$ Cognitive Psychology Unit, Institute of Psychology, Leiden University, the Netherlands \\ ${ }^{3}$ Department of Psychology, University of Amsterdam, the Netherlands \\ ${ }^{4}$ Psychology, School of Social Sciences, University of Dundee, Scotland \\ ${ }^{5}$ Cognitive \& Affective Psychophysiology Laboratory (CAPLAB), Department of Experimental \\ Clinical \& Health Psychology, Ghent University, Ghent, Belgium
}

\section{Corresponding author:}

Henk van Steenbergen

Wassenaarseweg 52

2333 AK Leiden

The Netherlands

Phone number: +31 (0)71 5273655

Fax number: +31 (0)71 5273783

E-mail address: HvanSteenbergen@fsw.leidenuniv.nl 


\section{Biographies}

\section{Henk van Steenbergen}

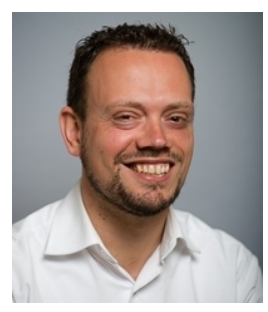

Institute of Psychology; Leiden University, Leiden, the Netherlands e-mail: HvanSteenbergen 0 ,fsw.leidenuniv.nl

Henk van Steenbergen is an assistant professor at Leiden University and also affiliated to the Leiden Institute for Brain and Cognition, the Netherlands. He received his $\mathrm{PhD}$ in cognitive neuroscience from Leiden University in 2012. In 2015 he received a tenured position as an assistant professor and started his Affect, Motivation, and Action lab.

Van Steenbergen studies the fascinating, yet intricate, link between emotion and cognition, focusing on the (mal)adaptive role of affective states in and on cognitive control, decision making and stress resilience in humans. He uses a multi-method approach that involves neuroimaging (EEG, MRI), physiology (pupil dilation, facial EMG and cardiovascular measures), pharmacological interventions, and computational modeling of behavior. He has also contributed methodological innovations to the field, including an online tool to run psychological experiments and he co-authored a book on the experiment builder software E-Prime. His most recent work has yielded pioneering studies in a new direction: the neurochemical opioid-mediated mechanisms that underlie the interactions between affect, cognitive control, and stress resilience. His work is funded by the Dutch Research Council (NWO) and by the interdisciplinary research program "Social Resilience and Security" of Leiden University. In 2020 he organized the Lorentz Center workshop on positive affect together with Siri Leknes (University of Oslo) and the other editors. This workshop was made possible in part by the support of the Leids Universiteits Fonds / Mr. J.J. van Walsem Fonds.

\section{Disa Sauter}

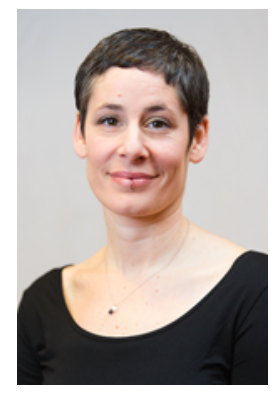

Department of Psychology, University of Amsterdam, the Netherlands e-mail: d.a.sauter@uva.nl

Disa Sauter is an Associate Professor in the Department of Psychology at the University of Amsterdam, the Netherlands. She did her BSc in Psychology and Cognitive Science at University College London (2002), followed by a PhD in the same department (2006). She then I worked as a post-doctoral researcher at the Institute of Psychiatry, 
King's College London, after which she received an ESRC fellowship, hosted at Birkbeck College London. In 2008, Sauter moved to the Netherlands to work as a staff researcher at the Max Planck Institute for Psycholinguistics, where she stayed until 2011. She has been at to the University of Amsterdam since 2011.

Sauter held a Veni grant (2011-2016) from the Dutch Science Foundation, and is current funded by an ERC Starting Grant and several grants from the Dutch Science Foundation. Sauter's work examines emotion with a particular focus on the communication of emotions via non-verbal expressions. She studies vocalisations like laughter, cries and sighs, and also facial expressions of emotions, with an interest in how factors such as preparedness, culture, and learning shape our emotions and the ways that they are communicated. Sauter's research makes use of a wide range of psychological methods, including cross-cultural comparisons, and she has a particular interest in positive emotions and distinctions between different positive affective states.

\section{Blair Saunders}

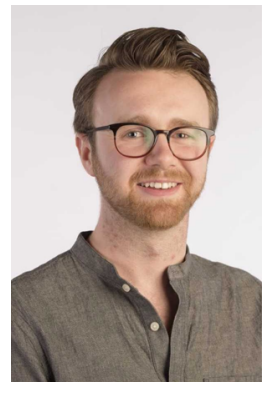

Psychology, School of Social Sciences, University of Dundee, Scotland

e-mail: b.z.saunders@dundee.ac.uk

Since 2017, Blair Saunders has worked as lecturer in Psychology at the University of Dundee where he started the Self-Regulation Laboratory. He received his $\mathrm{PhD}$ on the topic of "mood-dependent changes in cognitive control" from the University of St Andrews in 2014, and spent time as a postdoctoral fellow at the University of Toronto between his graduate research and starting his faculty position.

Saunders' research focuses on the integration of self-regulation and emotion. This indicates how variation in the implementation of selfregulation can be viewed as an instance of emotion regulation that aims to avoid unpleasant cognitive states brought about by cognitive conflict, unwanted desires, and self-generated mistakes. He draws evidence from multiple levels of analysis to achieve these goals, including behavioural, phenomenological, and psychophysiological (EEG, EMG) analyses. His work has been funded by the British Academy/Leverhulme Trust and the Carnegie Trust for the Universities of Scotland. 


\section{Gilles Pourtois}

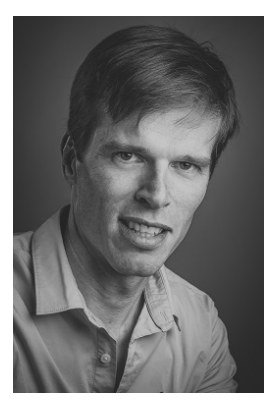

Cognitive \& Affective Psychophysiology Laboratory (CAPLAB); Department of Experimental Clinical \& Health Psychology; Ghent University; Ghent, Belgium

e-mail: gilles.pourtois@ugent.be

Gilles Pourtois is professor in psychology at Ghent University. He completed his $\mathrm{PhD}$ in cognitive neuroscience in 2002 at Tilburg University (The Netherlands). After his $\mathrm{PhD}$, he carried out postdocotral trainings in Nijmegen (Donders Institute) and Geneva (Swiss Center for Affective Sciences), before he moved to Ghent in 2008, where he was appointed associate research professor, and in 2017, full professor. In 2016, he founded the Cognitive \& Affective Psychophysiology Laboratory at Ghent University.

His research focuses on reciprocal interaction effects between cognition and emotion. He uses psychophysiological methods, including EEG. This research seeks to bridge the gap between experimental psychology and clinical psychology by generating and testing novel hypotheses regarding the nature and extent of affective influences on cognition in pathological conditions (anxiety and depression). More recently, he has explored effets of positive affect on performance monitoring. His work has been funded by the European Research Council (starting grant), the Research Foundation Flanders (FWO), the Special Research Fund from Ghent University, as well as the Brain \& Behavior Research Foundation (NARSAD Independent Investigator Grant). 


\section{Introduction}

Positive emotions are an important part of human life. They can be very strong, for example when we fall in love, land our dream job, or attend a concert or sports event. But more often they are less intense, for example when enjoying a meal or watching a sunset on a beautiful summer evening. Since the seminal work in the 1990s by Barbara Fredrickson, Martin Seligman and others in the 1990s, scientific research has made increasingly clear that positive affective states are not just mere epiphenomena but that they may have clear functional roles that are strongly rooted in our biological makeup. This special issue focuses on positive affect, an umbrella term that contains both specific emotions, such as gratitude, pride, and interest, as well as more long-term and diffuse affective states, such as happiness and content moods. For example, empirical research has shown that positive affect plays a role in behavioural flexibility, reducing stress, and facilitating psychosocial functioning. Recent work further indicates that understanding disturbances of positive affect can aid the diagnosis and treatment of mental disorders. However, despite the growing appreciation of the importance of positive affect in the fields of psychology and neuroscience, many questions still remain: What are positive emotions, how are they generated in the brain, how do they relate to and influence cognition, and how can this knowledge can be applied to improve mental and physical health, and well-being?

In March 2020, the editors of this special issue together with Siri Leknes (University of Oslo), brought a small group of researchers together in Leiden, the Netherlands to study these questions. They included emotion scientists, social and cognitive psychologists, and neuroscientists, each approaching positive affect from their own discipline's perspective. At the Lorentz Center we learned about each other's work and engaged in intense discussions with the aim to achieve cross-fertilization in this area. The insights gained yielded many of the papers in this special issue. Moreover, the event initiated several papers in which participants from different fields joined forces to produce interdisciplinary contributions to this special issue. In addition, we invited other experts and leaders in the field to contribute. Together, this results in a rich overview of research on positive affect, with contributions from a variety of different perspectives in psychology and neuroscience.

The current issue consists of 34 contributions that are divided into three overarching topics. Part I is concerned with the question of what constitutes positive affect and emotion. This section includes papers that introduce possible taxonomies of positive affect, and that differentiate between types of positive emotions and positive affective states, as well as their different behavioral and social functions. Part II focuses on mechanisms that generate or involve positive affect, identifying those at multiple levels of the brain, the body and behaviour, and showing how positive affect relates to and influences cognition and motivation. Finally, part III focuses on papers that describe applications of positive affect research in the domains of stress, health, and psychopathology.

\section{Part I. Taxonomies}

\section{What constitutes positive affect and emotion?}

The first paper considers the question of how to conceptualise positive emotion space; what constitutes a good taxology of positive emotions? Desmet, Sauter, and Shiota [1] introduce three key evaluative criteria against which typologies can be evaluated: comprehensiveness, distinctiveness, and granularity. Rather than defending one particular typology, they propose that 
these criteria can guide scholars in selecting the typology that best suits their needs. The contribution of Shiota, Sauter, and Desmet [2] also highlights that there are multiple ways to approach the question of what constitutes positive affect and emotion, focusing on how to define the key constructs themselves. Shiota and colleagues highlight three types of positivity that often coincide in lived emotional experience, but note that there are examples of divergence and dynamic ways in which they influence each other. They emphasize the importance of selecting and operationalizing the target construct with care in both basic affective science and translational research.

Traditional research in emotion science has often focused on the individual that experiences emotions. Brown and Fredrickson [3] instead introduce a new concept by focusing on co-experienced positive affective states, moments in which positive affect co-occurs in individuals who are engaged with one another via real-time sensory connection. Their paper shows that coexperienced positive affect encompasses the joint subjective experience, co-expression, and coembodiment of positive affect, and that this plays a critical role in the development of social skills, social bonds, and caring within communities.

Affective states often result in response to goals and motives, and can even become a goal in their own right. Moors, Van de Cruys, and Pourtois [4] compare existing theories that have taken goals into account. They contrast predictions from appraisal theories, goal-directed theories, and predictive processing theories, and outline how these theories differ in terms of parsimony and scope. Zerwas and Ford [5] zoom in on the situation when happiness becomes a goal in itself. They elaborate on research on the happiness paradox, where increased valuation of happiness is associated with lower rather than higher actual happiness, and show how a cybernetic model predicts different outcomes depending on the particular stage of goal pursuit.

Mercadante, Witkower, and Tracy [6] focus on one specific emotion, pride, highlighting two distinct facets, namely authentic pride and hubristic pride. Their paper is a good example of how a rich understanding of one specific emotion can in turn inform research into other positive emotions.

\section{Functional accounts}

Consistent with evolution theory, different positive states can serve various social and cognitive functions. Several recent taxonomies have started to map out these divergent functions. Revord, Sweeny, and Lyubomirsky [7] provide an overview of how contemporary emotion science approaches the function of positive emotions. They highlight that people can only experience emotions during specific episodes, which involve a specific eliciting situation, time frame, and cultural context, as well as an individual with his or her own idiosyncrasies. They argue that these contextual features are important for identifying the functions of discrete emotions. In their contribution, Keltner and Cowan [8] integrate top-down confirmatory studies of emotion and datadriven, large-scale computational studies. They outline the evidence for a taxonomy of eleven positive emotions and show how a taxonomy of positive emotions based on their social function will help advance the field of emotion science. Sels, Tran, Greenaway, Verhofstadt, and Kalokerinos [9] also focus on the social functions of positive emotions, with a particular emphasis on emotional expressions. They highlight three central functions of positive emotions including intimacy, impression, and influence. 
Gable and Dreisbach [10] address the different cognitive functions of positive affect and approach motivation. They provide a functional account of positive states in which positive affect associated with high motivational intensity promotes the narrowing of cognitive focus. Conversely, less motivationally intense positive affect might act as a safety signal that allows for increased flexibility and consideration of alternative goals.

\section{Part II. Mechanisms}

\section{Mechanisms at multiple levels}

Positive affect involves many components including appraisal, expression and subjective experience. These processes involve complex brain mechanisms in interaction with the body, and many contributions in this special issue show that a complete understanding of positive affective states requires multiple levels of analysis. Harmon-Jones and Harmon-Jones [11] emphasise that measurement error and biases can occur at each of these levels. They show that even the commonly used self-report measure of positive affect, the PANAS, might not measure the construct it is supposed to measure. They also discuss evidence showing that positive affect and approach motivation are not invariably positively associated [see also 10,12-14], an observation that has implications for the definition, operationalization, and measurement of positive affect.

Although many contributions to this special issue cover in more or less detail the neurobiology of positive states, several contributions focus directly on the neural underpinnings of emotion. Several papers focus on primary or secondary rewards, bridging human and nonhuman neuroscience. Nguyen, Naffziger, and Berridge [15] provide an update on recent animal neuroscience research on pleasure (or 'liking'), motivation (or 'wanting'), and reward-related learning. They show how liking and wanting can be dissociated in animal studies by measuring and manipulating activity in hedonic hotspots deep in the brain. Sander and Nummenmaa [16] discuss the emotional nature of rewards. They show that combining insights from appraisal theory and recent neural data can help to formulate an integrated approach that helps to describe the multifaceted emotional aspects of rewards at multiple levels of analysis. The paper by Blain and Sharot [17] focuses on cognitive and neural mechanisms suggesting that intrinsic reward stems from an increased sense of self-efficacy. According to this hypothesis, an activity is deemed intrinsically rewarding when the belief of self-efficacy is strengthened or promoted in the agent; they review recent neurobiological evidence linking self-efficacy to neural reward system activity.

Affective brain processes can also be studied using electroencephalography (EEG) measures over the scalp. In their contribution, Gable, Paul, Pourtois, and Burgdorf [12] review recent human electroencephalography measures of brain activity related to positive affect. They show how these measures in both the frequency domain (prefrontal alpha and premotor beta oscillations) and the time domain (reward positivity) provide insights into approach motivation and reward processing.

Positive emotions often involve the body, not only to express feeling states but also as a source of stimulation. Focusing on consummatory sensory pleasures, Nummenmaa and van Dillen [18] highlight the distinct mechanisms associated with sex, food, and social touch. Their work shows the distinct profiles different pleasures can have, and the need for multi-method approaches involving neuroimaging, psychophysiology, self-report, and endocrinological measures. 
Recent work has also started to integrate neuroeconomic and computational modeling to understand the neurocomputational processes that might underlie affective experiences. Using such an approach, Eldar, Pessiglione, and van Dillen [19] propose a computational mechanism in which happiness signals contexts in which rewards are becoming generally more available. The extent to which rewards are surprising generates happiness, and this differentiates happiness from other states related to reward consumption and subjective value such as pleasure. Foster Vander Elst, Vuust, and Kringelbach [20] apply a predictive coding framework to detail the different hedonic processes or cycles involved while listening to or processing (pleasant) music [see also 21]. They also consider action-related components such as groove and dance and how collective experiences resulting from music-making and dance increase social cohesion.

\section{Influence of positive affect on cognition}

Emotional and cognitive processes are often intertwined, perhaps even to the extent that they cannot be fully separated. Affective states therefore cannot be studied in isolation from cognition. Indeed, accumulating work has shown that emotion influences cognition which in turn can shape emotion, suggesting complex reciprocal interactions between these domains. Several contributions in this special issue showcase recent work on how positive affect influences cognitive and motivational processes. Chiew [22] provides an update on modulatory effects of positive affect on proactive and reactive control, including neuromodulatory mechanisms. She discusses the notion that positive affect and reward can have a variety of different effects on cognitive control, depending on the exact task demands. Paul, Pourtois, van Steenbergen, Gable, and Dreisbach [14] present and discuss recent research where the influence of positive affect and approach motivation on cognitive flexibility has been investigated, with a focus on cognitive and attentional control. Based on the similarities observed, their integrated account proposes that positive affect might increase flexibility and broadens attention under conditions of low levels of approach motivation, whereas high approach motivation could increase goal maintenance and narrow attention.

Lacey, Wilhelm, and Gable [13] further elaborate on the critical role of motivational intensity in the moderation of the relationship between positive affect and attentional scope. These authors also highlight the bidirectionality of these findings, such that attentional scope can drive changes in motivational intensity, and document empirical evidence in favour of this motivational account from diverse areas and across behavioural and neuroscientific levels of analysis.

\section{Positive affect and value as a central component in other processes}

Given the intertwined nature of emotion, cognition, and motivation, positive affect plays a role in many cognitive and social processes, although those are often overlooked in affective science. For example, Gruber and Fandakova [23] focus on how positive states, such as curiosity and surprise, might enhance memory through the developmental maturation of the hippocampus, prefrontal cortex, and the anterior cingulate cortex. Wuensch, Pool, and Sander [24] also discuss memory processes, focussing on learning stimulus-response associations that have acquired positive value. They suggest modeling individual differences in Pavlovian learning in order to reveal different learning phenotypes in human populations, with potential for translational and transdiagnostic insights.

The contribution by Dixon and Gross [25] focuses on the neural representation of the self. They propose that the self is organized into a network, consisting of interacting positive and 
negative self-belief units that are activated by positive and negative events. They show how differences in network organization eventually also shape affective experience and how this has implications for treatment of clinical disorders.

Ferguson, Cameron, and Inzlicht [26] focus on the role of pleasant feelings when people engage in empathic, pro-social behavior. They review recent work suggesting that it is not the act of being empathic itself that feels good. Rather empathy acquires positive value because humans associate it with the rewards that typically follow from empathic behaviour. Social behaviour is also the topic of the paper by Roth, Samara, Tan, Prochazkova, and Kret [27]. They provide a framework that integrates human and non-human findings regarding inter-individual coordination at the behavioural and physiological level, which they suggest is associated with improved pair bonding and heightened reproductive success in socially monogamous species.

Many enjoyable activities are typically carried out in a social context, and some of them might actually be pleasant because they have a social origin. Nummenmaa, Putkinen, and Sams [21] apply this idea to the domain of music-making and listening. They propose a "simulated sociability" hypothesis, according to which the social dimension of music is key for the pleasant feelings humans typically experience when listening to or making music. Consistent with this proposal, the authors summarize evidence from neural activity and neurochemistry showing substantial overlap between social stimuli and music.

\section{Part III. Applications}

\section{Stress and health}

Several contributions to this special issue aim to bridge the gap between theory and practice. Some of them focus on the beneficial role of positive affect in reducing stress, increasing resilience, and promoting healthy behaviour. The review by van Steenbergen, de Bruijn, van Duijvenvoorde, and van Harmelen [28] shows how positive affect can have stress-buffering effects in the brain, the body and behavior at multiple timescales, ranging from micro-stressors in cognitive tasks to stressors in daily life. They also highlight the neural and neurochemical systems that support these buffering effects.

Shiota, Papies, Preston, and Sauter [29] review both theoretical proposals and empirical evidence suggesting that positive affect-based interventions can be effective for promoting desirable behaviour change. They provide examples of such efforts in the domains of health behaviour, prosocial behaviour, and environmentally sustainable behaviour. Different mechanisms by which positive affect may help promote behaviour change are considered, and implications for future intervention development are discussed.

\section{Psychopathology}

Although most studies on psychopathology have focussed on negative affect and treatment, research on positive affect and prevention is growing rapidly. It is important to understand dysregulated positive affect in clinical samples, both to aid diagnosis and improve existing treatments and to develop new interventions. Villanueva, Silton, Heller, Barch, and Gruber [30] reflect on the current state of this field and outline seven challenges, with a focus on substance use. Heininga and Kuppens [31] focus on recent findings from ecological momentary assessment research investigating positive affect in relation to mood disorders. Using this method, fluctuations 
in positive affect can be recorded, modelled, and related to specific daily-life events. They specifically focus on the mood-brightening effect in individuals with mood disorders.

Training compassion and mindfulness might help to improve available psychotherapeutic interventions. In their contribution, Förster and Kanske [32] highlight recent data showing that affective states quickly change in response to brief periods of compassion training in healthy individuals, and that this involves plasticity of neural systems associated with care and soothing. Garland [33] presents a neurobiologically-grounded model of mindfulness, in which savouring the hedonic pleasure derived from natural rewards, self-generating interoceptive reward responses, and cultivating self-transcendent meaning are key elements. He suggests that mindfulness-based therapy can target and improve dysregulated hedonic processes in addiction.

Finally, Perini, Zetterqvist, and Mayo [34] zoom in on the relevance of altered positive affective processing in individuals engaging in nonsuicidal self-injury. Going beyond earlier work that has emphasized the role of negative affect and relief in these patient groups, they highlight initial evidence that suggests that nonsuicidal self-injury might also involve increased reactivity to positive stimuli.

\section{Conclusions}

As is evident from this summary, this special issue brings together a wide variety of methods and perspectives, thus providing a rich and up-to-date overview of positive affect research from prominent scholars in the field. Given its large and multidisciplinary scope, we believe that this issue is not only relevant for researchers who already work in the field, but that it will also help to familiarize students, scholars and practitioners who are interested in finding out about new developments in this area of work. We hope that this collection of articles will not only foster additional research into positive affect, but that it will also help to inspire new research directions and new collaborations on positive affect. 


\section{Literature}

1. Desmet PMA, Sauter DA, Shiota MN: Apples and oranges: Three criteria for positive emotion typologies. Current Opinion in Behavioral Sciences 2021, 39:119-124.

2. Shiota M, Sauter DA, Desmet PMA: What are "positive" affect and emotion? Current Opinion in Behavioral Sciences 2021, 39:142-146.

3. Brown CL, Fredrickson BL: Characteristics and consequences of co-experienced positive affect: understanding the origins of social skills, social bonds, and caring, healthy communities. Current Opinion in Behavioral Sciences 2021, 39:58-63.

4. Moors A, Van de Cruys S, Pourtois G: Comparison of the determinants for positive and negative affect proposed by appraisal theories, goal-directed theories, and predictive processing theories. Current Opinion in Behavioral Sciences 2021, 39:147-152.

5. Zerwas FK, Ford BQ: The paradox of pursuing happiness. Current Opinion in Behavioral Sciences 2021, 39:106-112.

6. Mercadante EJ, Witkower Z, Tracy J: The psychological structure, social consequences, function, and expression of pride experiences. Current Opinion in Behavioral Sciences 2021, 39:130-135.

7. Revord J, Sweeny K, Lyubomirsky S: Categorizing the function of positive emotions. Current Opinion in Behavioral Sciences 2021, 39:93-97.

8. Keltner D, Cowan WB: A taxonomy of positive emotions. Current Opinion in Behavioral Sciences 2021, 39.

9. Sels L, Tran A, Greenaway KH, Verhofstadt L, Kalokerinos EK: The social functions of positive emotions. Current Opinion in Behavioral Sciences 2021, 39:41-45.

10. Gable P, Dreisbach G: Approach motivation and positive affect. Current Opinion in Behavioral Sciences 2021, 39.

11. Harmon-Jones E, Harmon-Jones C: On defining positive affect (PA): considering attitudes toward emotions, measures of PA, and approach motivation. Current Opinion in Behavioral Sciences 2021, 39:46-51.

12. Gable P, Paul K, Pourtois G, Burgdorf J: Utilizing electroencephalography (EEG) to investigate positive affect. Current Opinion in Behavioral Sciences 2021, 39.

13. Lacey MF, Wilhelm RA, Gable PA: What is it about positive affect that alters attentional scope? Current Opinion in Behavioral Sciences 2021, 39:185-189.

14. Paul K, Pourtois G, van Steenbergen H, Gable P, Dreisbach G: Finding a balance: Modulatory effects of positive affect on attentional and cognitive control. Current Opinion in Behavioral Sciences 2021, 39:136-141. 
15. Nguyen D, Naffziger EE, Berridge KC: Positive affect: nature and brain bases of liking and wanting. Current Opinion in Behavioral Sciences 2021, 39:72-78.

16. Sander D, Nummenmaa L: Reward and emotion in the brain. Current Opinion in Behavioral Sciences 2021, 39:161-167.

17. Blain B, Sharot T: Intrinsic reward: potential cognitive and neural mechanisms. Current Opinion in Behavioral Sciences 2021, 39:113-118.

18. Nummenmaa L, van Dillen L: Carnal pleasures. Current Opinion in Behavioral Sciences 2021, 39:85-92.

19. Eldar E, Pessiglione M, van Dillen L: Positive affect as a computational mechanism. Current Opinion in Behavioral Sciences 2021, 39:52-57.

20. Foster Vander Elst O, Vuust P, Kringelbach ML: Sweet anticipation and positive emotions in music, groove, and dance. Current Opinion in Behavioral Sciences 2021, 39:79-84.

21. Nummenmaa L, Putkinen V, Sams M: Social pleasures of music. Current Opinion in Behavioral Sciences 2021, 39.

22. Chiew KS: Revisiting positive affect and reward influences on cognitive control. Current Opinion in Behavioral Sciences 2021, 39:27-33.

23. Gruber M, Fandakova Y: Curiosity in childhood and adolescence: What can we learn from the brain. Current Opinion in Behavioral Sciences 2021, 39:178-184.

24. Wuensch L, Pool ER, Sander D: Individual differences in learning positive affective value. Current Opinion in Behavioral Sciences 2021, 39:19-26.

25. Dixon ML, Gross JJ: Dynamic network organization of the self: implications for affective experience. Current Opinion in Behavioral Sciences 2021, 39:1-9.

26. Ferguson AM, Cameron CD, Inzlicht M: When does empathy feel good? Current Opinion in Behavioral Sciences 2021, 39:125-129.

27. Roth TS, Samara I, Tan J, Prochazkova E, Kret ME: A comparative framework of interindividual coordination and pair-bonding. Current Opinion in Behavioral Sciences 2021, 39:98-105.

28. van Steenbergen H, de Bruijn ERA, van Duijvenvoorde ACK, van Harmelen A-L: How positive affect buffers stress responses. Current Opinion in Behavioral Sciences 2021, 39:153-160.

29. Shiota M, Papies EK, Preston SD, Sauter DA: Positive affect and behaviour change. Current Opinion in Behavioral Sciences 2021, 39. 
30. Villanueva CM, Silton RL, Heller W, Barch DM, Gruber J: Change is on the horizon: call to action for the study of positive emotion and reward in psychopathology. Current Opinion in Behavioral Sciences 2021, 39:34-40.

31. Heininga VE, Kuppens P: Psychopathology and positive emotions in daily life. Current Opinion in Behavioral Sciences 2021, 39:10-18.

32. Förster K, Kanske P: Exploiting the plasticity of compassion to improve psychotherapy. Current Opinion in Behavioral Sciences 2021, 39:64-71.

33. Garland E: Mindful positive emotion regulation as a treatment for addiction from hedonic pleasure to self-transcendent meaning. Current Opinion in Behavioral Sciences 2021, 39:168-177.

34. Perini I, Zetterqvist M, Mayo LM: Beyond distress: A role for positive affect in nonsuicidal self-injury. Current Opinion in Behavioral Sciences 2021, 39. 